\title{
Rectal Cancer Patient with Complete Response to Neoadjuvant Chemoradiotherapy
}

\author{
Seung-Gu Yeo \\ Department of Radiation Oncology, Soonchunhyang University Cheonan Hospital, Soonchunhyang University College of Medicine, Cheonan, Korea
}

\begin{abstract}
Personalized medicine for rectal cancer patients includes nonoperative management. This strategy is appropriate for select patients who exhibit a clinical complete response ( $(C R)$ to neoadjuvant chemoradiotherapy ( $\mathrm{nCRT}$ ). This study presents the case of a 70-year-old female with locally advanced rectal cancer. She received standard nCRT, consisting of 50.4 Gy pelvic radiotherapy and concurrent 5-fluorouracil chemotherapy. The response to nCRT was evaluated at 11 weeks after nCRT completion. Colonoscopy and computed tomography indicated CCR, but a partial residual tumor was suspected based on magnetic resonance imaging. Low anterior resection was performed with the creation of a protective colostomy. The pathological examination of the surgical specimen revealed only a fibrotic mass, no viable tumor cells. The stoma was maintained for 10 months until reversal after adjuvant chemotherapy ended. The patient remains alive with no evidence of disease 4 years later; however, the utility of this radical major surgery was questionable for this patient. Development of a risk-adapted strategy based on the nCRT response would avoid overtreatment, improving functional outcomes and quality of life while also retaining good oncological outcomes.
\end{abstract}

Keywords: Rectal neoplasms; Non-operative management; Watchful waiting; Radiotherapy; Complete regression; Case reports

\section{INTRODUCTION}

Standard management for patients with locally advanced rectal cancer (LARC) includes neoadjuvant chemoradiotherapy (nCRT), radical surgery, and adjuvant chemotherapy. However, LARC patients are not a homogenous group in terms of their responses to nCRT. At one end of this response, the spectrum is a complete tumor response [1]. A pathological complete response (pCR) confirmed via surgical pathology has been reported in about $20 \%$ of LARC patients after the standard nCRT regimen [2]. For this subgroup, radical surgery plays a limited role in identifying pCR, and removes no cancer cells. Surgery may thus not be justified, considering the risks of mortality and morbidity.

This study presents the case of an LARC patient who is alive with no evidence of disease at 4 years after receiving all standard multimodal treatments. However, residual tumor cells were not found in the surgical specimen obtained via radical resection combined with the protective colostomy.

\section{CASE REPORT}

The patient was a 70-year-old female. Her rectal cancer was diagnosed in the absence of specific symptoms during a routine medical check-up performed in March 2015. Colonoscopy revealed an ulcerofungating mass 5 to $8 \mathrm{~cm}$ from the anal verge (Fig. 1A). The pathological examination of a biopsy specimen revealed a well-differentiated adenocarcinoma. The serum level of carcinoembryonic antigen was $3.77 \mathrm{ng} / \mathrm{mL}$ (normal range, 0-5.0 ng/mL). Abdominopelvic computed tomography (CT) and pelvic magnetic resonance imaging (MRI) revealed circumferential wall thickening with perirectal fat infiltration (Fig. 1B, D). Several small lymph nodes were evident in the mesorectum and presacral area. The clinical stage was determined to be T3N2aM0 according to the "AJCC cancer staging manual," 7th edition. Written informed consent was obtained from the patient.

To simulate neoadjuvant radiotherapy, the patient was immobilized in the prone position using a belly board. A contrast-en- 

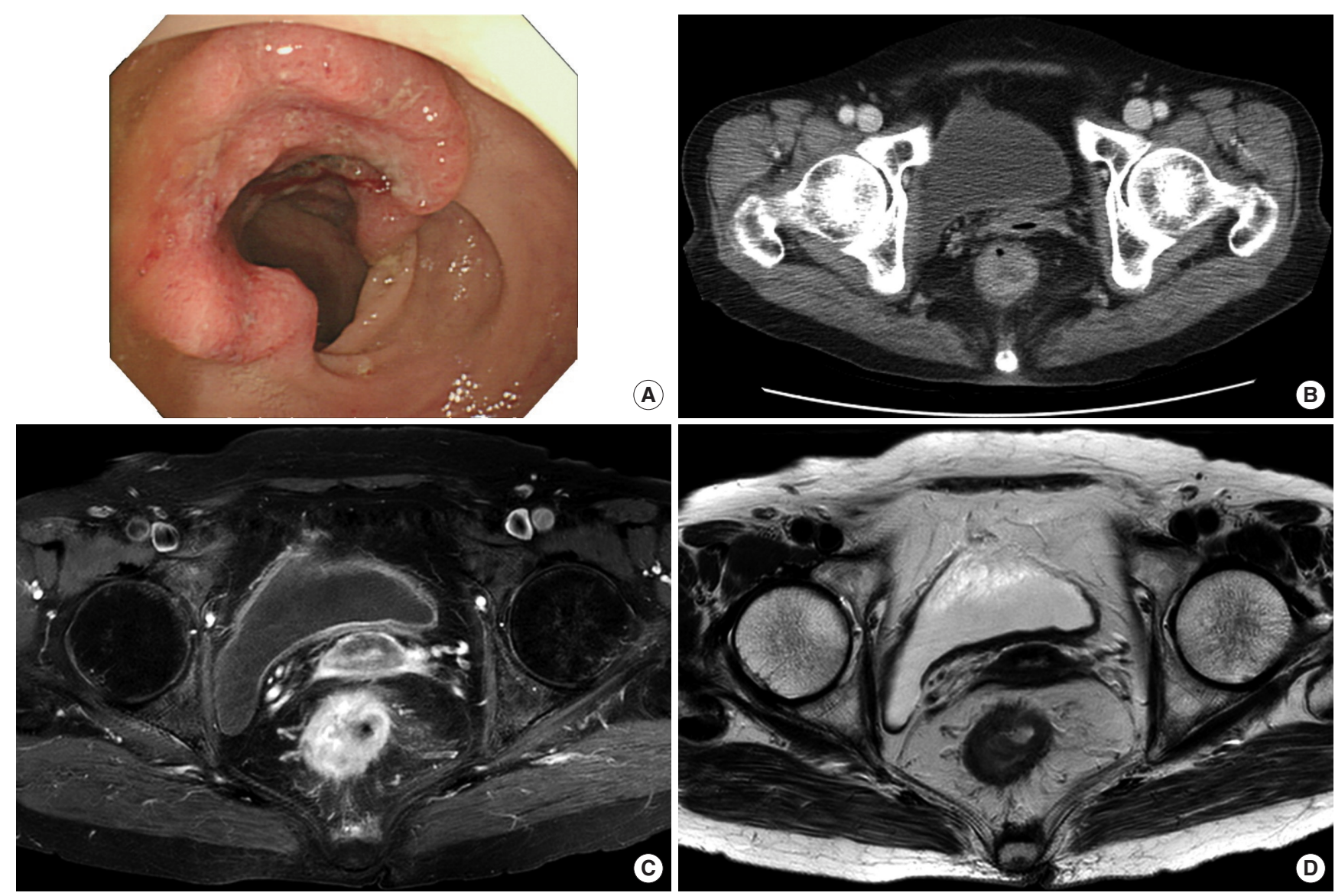

Fig. 1. Evaluation at presentation. (A) Colonoscopy. (B) Computed tomography. (C, D) Magnetic resonance images: gadolinium-enhanced T1 weighted with fat suppression (C) and T2 weighted without fat suppression (D).

hanced planning CT scan was performed using a 16-slice CT scanner (Brilliance CT Big Bore; Philips Medical Systems, Cleveland, $\mathrm{OH}, \mathrm{USA})$. Axial CT images were imported into the Eclipse RT planning system (Varian Medical Systems Inc., Palo Alto, CA, USA). The clinical target volume encompassed the gross mural tumor, involved lymph nodes, mesorectum, presacral space, and the internal iliac and distal common iliac lymphatics. The planning target volume was generated by adding a $5-\mathrm{mm}$ isotropic setup margin around the clinical target volume. The three dimensional conformal radiotherapy plan featured opposed $15 \mathrm{MV}$-photon opposed lateral fields with a wedge of $45^{\circ}$, and a $6 \mathrm{MV}$-photon posterior anterior field. The plan was normalized to ensure that $\geq 95 \%$ of the planned target volume received $100 \%$ of the prescription dose. Radiotherapy (50.4 Gy in 28 fractions) was delivered by a Novalis Tx system (Varian Medical Systems and Brainlab, Feldkirchen, Germany). Concurrent chemotherapy featured two cycles of bolus infusion of 5-fluorouracil ( $450 \mathrm{mg} / \mathrm{m}^{2} /$ day) and leu- covorin ( $20 \mathrm{mg} / \mathrm{m}^{2} /$ day) for 5 days between fractions $1-5$ and $24-$ 28 of radiotherapy. nCRT was completed without interruption and in the absence of any severe complications.

Evaluation of the nCRT response was scheduled for 6 weeks later but was postponed for reasons personal to the patient. Assessment was performed at 11 weeks after nCRT completion. No mass or stenosis was palpable in a digital rectal examination. Bluish venous dilatation, but no intraluminal mass or ulceration, was evident in colonoscopy (Fig. 2A). A CT scan revealed no definite mass lesion or wall thickening of the rectum (Fig. 2B). MRI scans indicated that the circumferential wall thickening was reduced but perhaps not eliminated (Fig. 2C, D). Low anterior resection with total mesorectal excision and a protective colostomy was performed at 80 days after nCRT completion. Surgical pathology revealed only a fibrotic mass with no viable tumor cells, indicative of tumor regression grade 4 [3]. The 18 lymph nodes removed were all negative. Six cycles of adjuvant chemotherapy using 5-fluorouracil and 

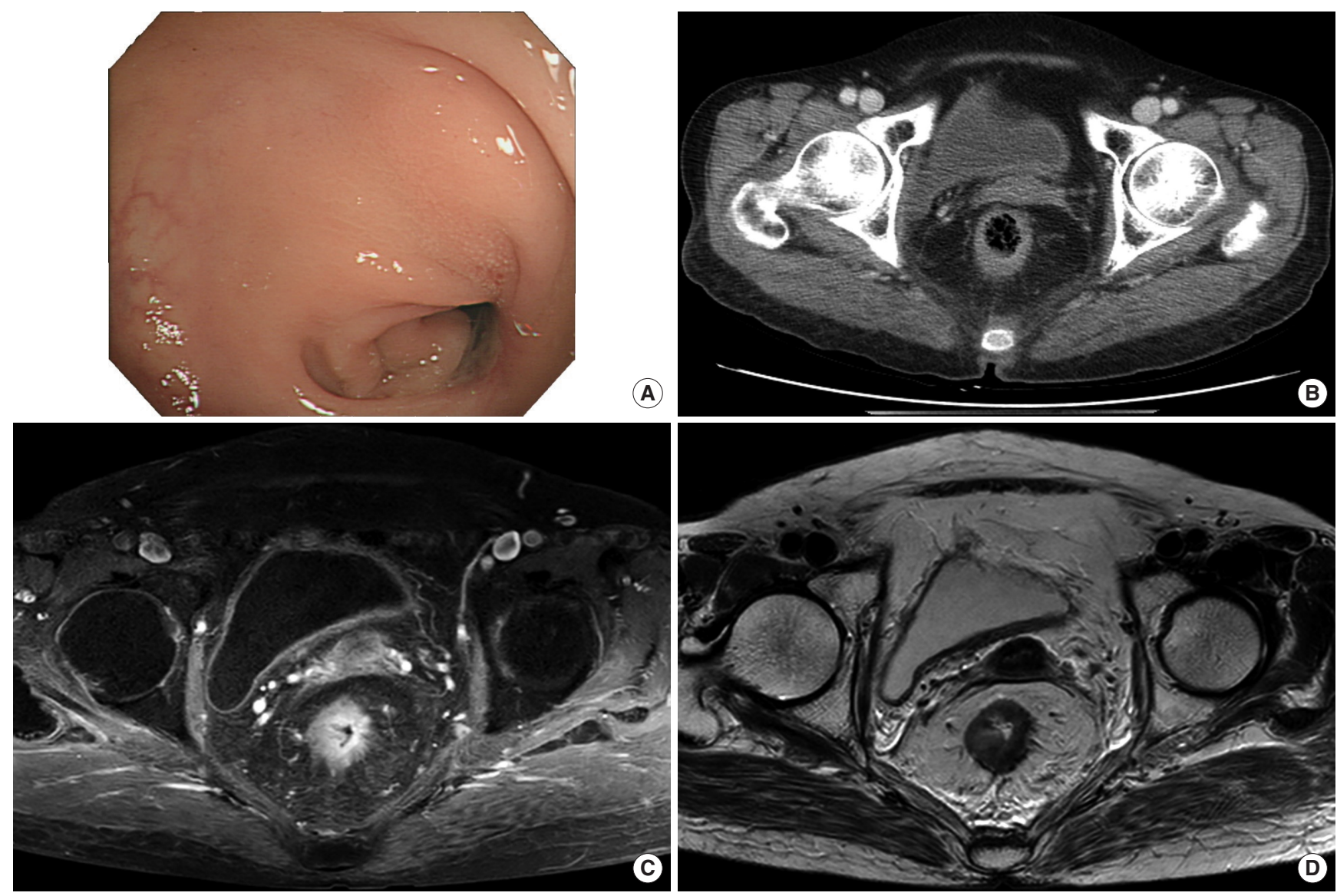

Fig. 2. Evaluation at 11 weeks after neoadjuvant chemoradiotherapy. (A) Colonoscopy. (B) Computed tomography. (C, D) Magnetic resonance images: gadolinium-enhanced T1 weighted with fat suppression (C) and T2 weighted without fat suppression (D).

leucovorin were given over 5 months. The temporary colostomy was reversed after completion of adjuvant chemotherapy.

Follow-up evaluation consisted of physical examinations, complete blood cell counts, liver function tests, and measurement of carcinoembryonic antigen levels every 3 months for the first 2 years and every 6 months thereafter. Chest radiography and abdominopelvic CT were performed every 6 months, and colonoscopy was done annually. Treatment toxicity featured frequent defecations and peristomal dermatological problems, which subsided with conservative management. No severe side effects were observed. The patient remains alive with no evidence of disease at 4 years later.

\section{DISCUSSION}

The patient had a good long-term oncological outcome. This favorable prognosis was predicted early when $\mathrm{pCR}$ was identified. A better CRT response is well known to be correlated with less aggressiveness of tumor and better prognosis of the patient [1]. LARCs of clinical stage II-III are newly stratified into pathological stages 0-III after nCRT. This new pathological stage is strongly associated with long-term outcomes [2]. This supports the suggestion that treatments should be de-intensified for a subgroup of LARC patients who exhibit a good tumor response to nCRT.

The standard surgery for LARC is an invasive procedure involving transabdominal radical resection: low anterior resection, proctectomy with total mesorectal excision and coloanal anastomosis, or abdominoperineal resection [4]. This operation is associated with a perioperative mortality of $1 \%-2 \%$, long-term morbidities such as urinary or sexual dysfunction and fecal incontinence, and may trigger a need for a temporary or permanent colostomy [5]. The anal structure was not sacrificed but a protective temporary colostomy was created and maintained for 10 months. Although no severe complications developed, the decision to per- 
form radical surgery is questionable in this patient, as it only identified pCR. A part of body organ was resected wherein all cancer cells had already been eradicated by the upfront CRT.

Nonoperative management (NOM), or watch-and-wait, has attracted increasing interest by clinicians managing rectal cancer patients who exhibit a clinical complete response (cCR) after nCRT. A series of reports on this strategy has been published by a Brazilian colorectal surgeon, Dr. Angelita Habr-Gama [6]. Recently, an international multicenter registry study reported the long-term outcomes of rectal cancer patients with cCR managed via NOM [7]. In total, 880 patients were analyzed over a median follow-up time of 3.3 years. The 2 -year cumulative incidence of local regrowth was $25.2 \%$; $88 \%$ of all local regrowth was diagnosed in the first 2 years and $97 \%$ of local regrowth was located in the bowel wall. Distant metastases were diagnosed in $8 \%$ of patients. Local unsalvageable disease after NOM was rare and long-term outcomes were excellent, with 5-year overall survival and diseasespecific survival rates of $85 \%$ and $94 \%$, respectively. The authors hypothesized that the risk for metastasis and cancer-specific death in such patients was more closely associated with tumor biology than the omission of immediate surgery.

If NOM is to be successful, it is critical to perform an accurate clinical evaluation of the CRT response. However, this remains challenging; the findings do not always correlate with the pathological evaluations [8]. In addition, the timing of nCRT response assessment is important. Several studies have suggested that longer interval periods are associated with increased rates of $\mathrm{CR}$, and it may take up to 12 weeks for CR rates to plateau after delivery of a standard nCRT regimen [9]. In the present case, the response was evaluated at 11 weeks after CRT completion. cCR status was predicted by endoscopic and CT evaluations, but the residual disease was suspected on MRI. The risk that MRI re-stages a patient inaccurately in terms of $\mathrm{pCR}$ prediction is much greater than the risk that occult cancer cells may exist in a surgical specimen reported being pCR [10]. Further deferral of surgery with frequent followups until one year would give this patient a sustained-CR status [6], and that major surgery and colostomy would have eventually been avoided.

Currently, NOM is not a recognized standard of care and thus should be applied only in clinical trials. However, in the near future, NOM may be recognized as an option for select patients whose rectal cancer is of cCR status after nCRT. This case study supports the need for personalized medicine, which will improve functional outcomes and quality of life while not compromising oncological outcomes.

\section{REFERENCES}

1. Yeo SG, Kim DY, Kim TH, Chang HJ, Oh JH, Park W, et al. Pathologic complete response of primary tumor following preoperative chemoradiotherapy for locally advanced rectal cancer: long-term outcomes and prognostic significance of pathologic nodal status (KROG 09-01). Ann Surg 2010;252:998-1004.

2. Yoo BC, Yeo SG. Clinical utility of pretreatment prediction of chemoradiotherapy response in rectal cancer: a review. EPMA J 2017;8:61-7.

3. Rodel C, Martus P, Papadoupolos T, Fuzesi L, Klimpfinger M, Fietkau R, et al. Prognostic significance of tumor regression after preoperative chemoradiotherapy for rectal cancer. J Clin Oncol 2005;23:8688-96.

4. Maurer CA, Renzulli P, Kull C, Kaser SA, Mazzucchelli L, Ulrich A, et al. The impact of the introduction of total mesorectal excision on local recurrence rate and survival in rectal cancer: long-term results. Ann Surg Oncol 2011;18:1899-906.

5. Paun BC, Cassie S, MacLean AR, Dixon E, Buie WD. Postoperative complications following surgery for rectal cancer. Ann Surg 2010;251:807-18.

6. Dattani M, Heald RJ, Goussous G, Broadhurst J, Sao Juliao GP, Habr-Gama A, et al. Oncological and survival outcomes in watch and wait patients with a clinical complete response after neoadjuvant chemoradiotherapy for rectal cancer: a systematic review and pooled analysis. Ann Surg 2018; 268:955-67.

7. Van der Valk MJ, Hilling DE, Bastiaannet E, Meershoek-Klein Kranenbarg E, Beets GL, Figueiredo NL, et al. Long-term outcomes of clinical complete responders after neoadjuvant treatment for rectal cancer in the International Watch \& Wait Database (IWWD): an international multicentre registry study. Lancet 2018;391:2537-45.

8. Smith FM, Wiland H, Mace A, Pai RK, Kalady MF. Clinical criteria underestimate complete pathological response in rectal cancer treated with neoadjuvant chemoradiotherapy. Dis Colon Rectum 2014;57:311-5.

9. Sloothaak DA, Geijsen DE, van Leersum NJ, Punt CJ, Buskens CJ, Bemelman WA, et al. Optimal time interval between neoadjuvant chemoradiotherapy and surgery for rectal cancer. Br J Surg 2013;100:933-9.

10. Ryan JE, Warrier SK, Lynch AC, Heriot AG. Assessing pathological complete response to neoadjuvant chemoradiotherapy in locally advanced rectal cancer: a systematic review. Colorectal Dis 2015;17:849-61. 